\section{Publicidade e representação social da Naturologia em jornais e revistas não acadêmicas no Brasil}

\section{Advertising and social representation of Naturology in newspapers and magazines in Brazil}

\section{RESUMO}

Objetivo: Identificar as representações sociais acerca da Naturologia divulgadas na mídia digital por jornais e revistas não acadêmicas no Brasil. Método: Pesquisa do tipo documental, realizada no acervo digital das revistas e jornais de maior circulação no Brasil. Os dados referentes à amostra de notícias foram processados no software IRAMUTEQ ${ }^{\circledR} \mathrm{e}$ analisados a partir da freqüência, ocorrência e similitude entre os termos. A análise foi feita a luz da teoria da representação social por meio dos mecanismos de ancoragem e objetivação. Resultados: Foi possível identificar que a naturologia é representada em 4 grandes grupos $(\mathrm{p}<0,0001)$ : formação profissional na área $(31,0 \%)$, regulamentação governamental (26,7\%), ensino de técnicas autoaplicáveis (25,4\%), e práticas em grupos específicos (16,9\%). Os assuntos são abordados objetivando apresentar a naturologia como uma opção extra ao alcance do bem-estar. Conclusão: As representações da mídia colaboraram na apresentação da naturologia como uma formação acadêmica cercada de regulamentos, o que é um aspecto positivo, porém ainda é forte o caráter complementar e com aspectos negativos como temas centrais. Assim, considerando a influência destas mídias, faz-se necessário maior aprofundamento acerca da representação social da Naturologia.

\section{PALAVRAS-CHAVE:}

Naturologia.

Percepção social.

Meios de comunicação.

Teoria das representações sociais.
Marcos Renato De Oliveira

- Universidade Federal do Piauí.

Faculdade de Enfermagem. Picos-PI. Brasil. Enfermeiro. Especialista em Saúde Pública - UECE. Mestre em Enfermagem na Promoção da Saúde - UFC. Doutor em Cuidados Clinicos em Enfermagem e Saúde - UECE.

Daniele Vasconcelos Fernandes Vieira

- Universidade Estadual do Ceará.

Faculdade de Medicina. Fortaleza-CE. Brasil. Enfermeira. Especialista em Terapias Holísticas e Complementares - IEDUCARE. Mestre em Cuidados Clinicos em Enfermagem e Saúde UECE.

Thatiana Araujo Maranhão

- Universidade Estadual do Piauí. Faculdade de Enfermagem. ParnaíbaPI. Brasil. Enfermeira. Especialista em Saúde da Família - UNINOVAFAPI. Mestre em Ciências da Saúde - UFPI. Doutora em Cuidados Clinicos em Enfermagem e Saúde - UECE

Raimundo Augusto Martins Torres Universidade Estadual do Ceará. Faculdade de Enfermagem. Fortaleza - CE. Brasil. Enfermeiro. Especialista em Educação Profissional - FIOCRUZ Mestre em Enfermagem na Promoção da Saúde - UFC. Doutor em Educação - UFC.

DOI: $10.19177 /$ cntc.v7e12201813-2

CORRESPONDENTE

Marcos Renato De Oliveira

Universidade Federal do Piauí - UFPI. Rua Ciçero Eduardo S/N. Picos, PI, Brasil, 64600000

E - M A I L

enfmarcosrenato@hotmail.com

Recebido: 26/07/2018

Aprovado: 13/09/2018 


\begin{abstract}
Objective: To identify the social representations about Naturology published in the digital media by newspapers and magazines in Brazil. Method: Documentary research, carried out in the digital collection of the most popular newspapers and magazines in Brazil. The data related to the news sample were processed in the IRAMUTEQ ${ }^{\circ}$ software and analyzed based on the frequency, occurrence and similarity between the terms. The analysis guided by the theory of social representation through the mechanisms of anchoring and objectification. Results: It was possible to identify that naturology is represented in 4 large groups $(\mathrm{p}<0.0001)$ : professional training in the area $(31.0 \%)$, government regulation $(26.7 \%)$, teaching of self-applied techniques \%), and practices in specific groups (16.9\%). Conclusion: The media representations have collaborated for the presentation of naturology as an academic formation surrounded by regulations, which is a positive aspect, but is very present the idea of a complementary practice and in negative aspects. Thus, considering the influence of these media, it is necessary to study more about the social representation of Naturology.
\end{abstract}

KEY WORDS: Naturology. Social perception. Media. Theory of social representations.

\section{INTRODUÇÃO}

A contemporaneidade é marcada por uma sociedade com vasto poder de acesso à informação, na qual a mídia exerce forte influência sobre todas as instâncias sociais, abrangendo inclusive os padrões de consumo na saúde. Nesse cenário, incluem-se interesses, instituições e atores sociais como partes integrantes do processo de engendramento das notícias, e, é preciso compreender como este agrupamento de interesses interfere na saúde humana ${ }^{1}$.

Durante o processo de procura por homeostase, o ser humano, tende a buscar orientação, seja por contato direto com seus pares, ou, como mais percebido atualmente, através da leitura de fontes mais formais, percebidas como confiáveis. Sendo os jornais e as revistas não acadêmicas, por sua vez, meios de informação cada vez mais acessíveis, e com a popularidade da rede mundial de computadores, estes são consumidos por todo o país.

Nesta busca por equilíbrio e bem estar, cada vez mais pessoas tem demonstrado interesse em práticas, que no Brasil são conhecidas como integrativas e complementares do cuidado, destacando-se as oriundas da naturologia.

A naturologia é considerada uma área do conhecimento das ciências da saúde e tem suas práticas orientadas no aumento da vitalidade do ser, ou das forças que produzem saúde, agregando os sistemas terapêuticos complexos, partindo de uma visão multidimensional do processo saúde-doença. Conforme as palavras de Sabbag² a naturologia é uma ciência na qual se utiliza da interagência de práticas integrativas e complementares no cuidado e atenção à saúde.

Sobre o foco da prática naturológica, descreve-se que a abordagem não está centrada apenas na aplicação de práticas naturais, mas que tem a matriz estabelecida em ações que restaurem a integridade e harmonia orgânica do que definem como interagente. Compreende-se que a naturologia não se reduz ao uso de práticas naturais dissociadas de princípios inerentes aos seus métodos e abordagens terapêuticas próprias, que se caracterizam como uma medicina não convencional, ampliada da visão tecnicista de cuidar a qual visa à implementação de técnicas e práticas com fins mercadológicos e imediatistas ${ }^{3}$.

O naturólogo se orienta pelo conhecimento oriundo das ciências biológicas, humanas e sociais que lhes confere uma abordagem terapêutica ampliada. Esse profissional contempla em suas práticas recursos como plantas medicinais, essências florais, argila medicinal, água em diferentes temperaturas, cores, toques através da massoterapia e da reflexoterapia, dentre muitos outros ${ }^{4-5}$. 
A participação do naturólogo no Sistema Único de Saúde (SUS), quanto ao espaço de visibilização pública, necessita de ampla divulgação por meio de pesquisas científicas, apresentação de projetos e ações aos gestores municipais de saúde, que possibilitem ampliar os serviços e a participação do naturólogo no SUS, além de registrar e divulgar as experiências executadas na rede pública municipal e estadual, organizar e fazer parte de grupos de estudos e pesquisas científicas nas universidades, bem como maior envolvimento dos naturólogos em ações coletivas para que a classe seja conhecida e reconhecida na sociedade ${ }^{5}$.

Entretanto, apesar de ser uma prática já bem difundida por todo o país, e de que grande parte da população esteja aberta a naturologia, ainda existe muita resistência, e pouco se sabe sobre a fonte desta resistência, inclusive por parte de alguns meios de comunicação.

Ainda, pouco se conhece sobre o poder de influencia das mídias informativas quanto a busca e o consumo das práticas de naturologia pelos usuários. Sendo necessário mais estudos para compreender a relação das noticias jornalísticas com a naturologia ${ }^{6}$.

$\mathrm{Na}$ década atual, a sociedade clama para que a mídia realize uma publicidade clara e coerente, levando em consideração inclusive o público que consome o conteúdo apresentado, pois já não existe somente uma leitura do conteúdo exposto, mas sim uma internalização que pode interferir inclusive nos padrões de saúde e doença, especialmente entre os sujeitos que ainda não compreendem bem os assuntos ora expostos ${ }^{7}$.

Diante do exposto, se questiona como a mídia textual brasileira apresenta a naturologia para o público geral. Assim, este estudo tem por objetivo identificar as representações sociais acerca da naturologia divulgados na mídia digital por jornais e revistas não acadêmicas no Brasil.

\section{MÉTODOS}

O estudo consiste em uma pesquisa do tipo documental, com abordagem capaz de extrair informações de fontes sem tratamento analítico como, jornais, revistas, vídeos, cartas, fotografias que, por sua vez, podem vir a ser meios de indicação e esclarecimento para elucidar determinadas questões sociais ${ }^{8-9}$.

Foram utilizadas neste estudo notícias veiculadas em revistas e jornais não acadêmicos publicados no Brasil. A amostra foi composta pelos seguintes meios de comunicação: revistas Veja, Exame, IstoÉ, os jornais Folha de São Paulo, Globo e Supernotícia. Estes veículos de informação foram selecionados devido ao número de publicações mensal em todo o país, sendo que estas mídias citadas abrangem a maioria do mercado da área no Brasil.

Os textos selecionados não tiveram limites de anos, pois assim seria feito uma melhor seleção das notícias, utilizando do próprio banco de dados disponíveis no Word Wide Web (WWW) da rede mundial de computadores de cada sítio eletrônico dos bancos supracitados.

A coleta de dados foi realizada no final do primeiro semestre de 2018. Utilizou-se como descritores de busca as seguintes palavras: naturologia e naturólogo. Como critérios de inclusão, selecionaram-se as notícias disponíveis na íntegra que versassem o tema pesquisado. Foram excluídas notícias apresentadas no campo de busca, mas que não faziam alusão ao tema buscado sendo, por exemplo, apresentadas por simples algoritmos de pesquisa com fins comerciais.

Após reunidos, os dados foram transcritos para o open software writer $\mathrm{e}$, em seguida, foram processados pelo IRAMUTEQ (Interface de R pour les Analyses Multidimensionnelles de Textes et de Questionnaires). Este software viabiliza diferentes tipos de análise de dados textuais, como a lexicografia básica, até análises multivariadas. $\mathrm{O}$ software apresenta rigor estatístico e permite aos pesquisadores utilizarem diferentes recursos técnicos de análise lexical ${ }^{10}$.

Para captar as representações sociais da mídia sobre a naturologia, foram empregadas análises oriundas do diagrama de Zipf, classificação hierárquica descendente (CHD), dendograma da CHD, análise de similitude, nuvem de palavras, especificidade e análise fatorial de correspondência (AFC). 
Os resultados obtidos também foram analisados ainda à luz da teoria das representações sociais por meio de dois sub-processos: a ancoragem e a objetivação. O primeiro buscou ancorar as ideias e assim reduzi-las a categoria. Já o segundo reproduziu um conceito em uma imagem identificando e explicitando a característica ícone àquela ideia ${ }^{11}$. Gráficos foram construídos no software Microsoft Office Excel 2007 para mostrar a ancoragem e a objetivação das reportagens.

O presente estudo não necessitou de parecer de comitê de ética, por analisar dados de livre acesso ao público, porém, respeitou-se os direitos autorais, $\mathrm{e}$ as, demais normas correlatas no Brasil foram seguidas, sendo que os conteúdos apresentados foram devidamente citados.

\section{RESULTADOS}

Após ampla busca, foram identificadas 78 publicações, das quais 9 foram excluídas por terem repetição, publicadas mais de uma vez por cada mídia, e 43 por não fazerem alusão direta ao tema. Assim, o corpus foi composto por 26 publicações, sendo que seus segmentos foram identificados a cada $40 \mathrm{li}$ nhas, resultando em 366 segmentos de texto. Além disso, identificou-se 12.624 ocorrências, 3.595 formas textuais e 2.227 hapax (17,6\% das ocorrências). O diagrama de Zipf apontou uma distribuição normal dos dados, o que indica que embora sejam provenientes de diferentes fontes, os dados foram homogêneos quanto à apresentação e distribuição do conteúdo investigado.

O Gráfico 1 demonstra a caracterização da temporalidade das publicações. E, observou-se que apesar de naturologia não ser um tema recente, somente apareceu em jornais e revistas de grande impacto nacional há 17 anos, ou, se apresentados antes disto, não constam nas bases online das fontes pesquisadas. Os anos de 2007 e 2008 foram os que apresentaram o maior número de publicações. Porém, se comparada com outros temas, a quantidade de publicações, ainda é baixa, com uma média de duas publicações por ano.
Gráfico 1 - Distribuição das publicações sobre naturologia nas mídias impressas nacionais. Brasil. 2018

Publicações por ano

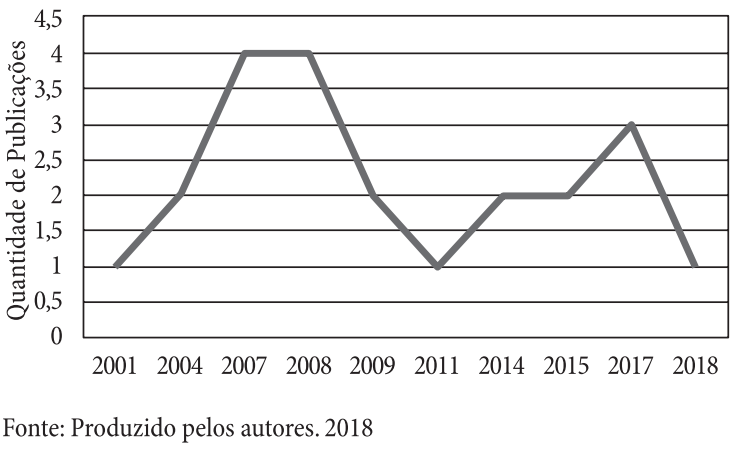

Por meio da observância da especificidade e análise fatorial de correspondência (AFC) foi possível identificar que a naturologia é representada em quatro grandes grupos $(\mathrm{p}<0,0001)$, a saber: formação profissional na área (31\%), regulamentação governamental (26,7\%), ensino de técnicas autoaplicáveis $(25,4 \%)$ e, práticas em grupos específicos $(16,9 \%)$. Conforme obsevado na imagem 1, de AFC.

Figura 1 - Análise fatorial de correspondência (AFC) sobre naturologia nas mídias impressas brasileiras. Brasil. 2018

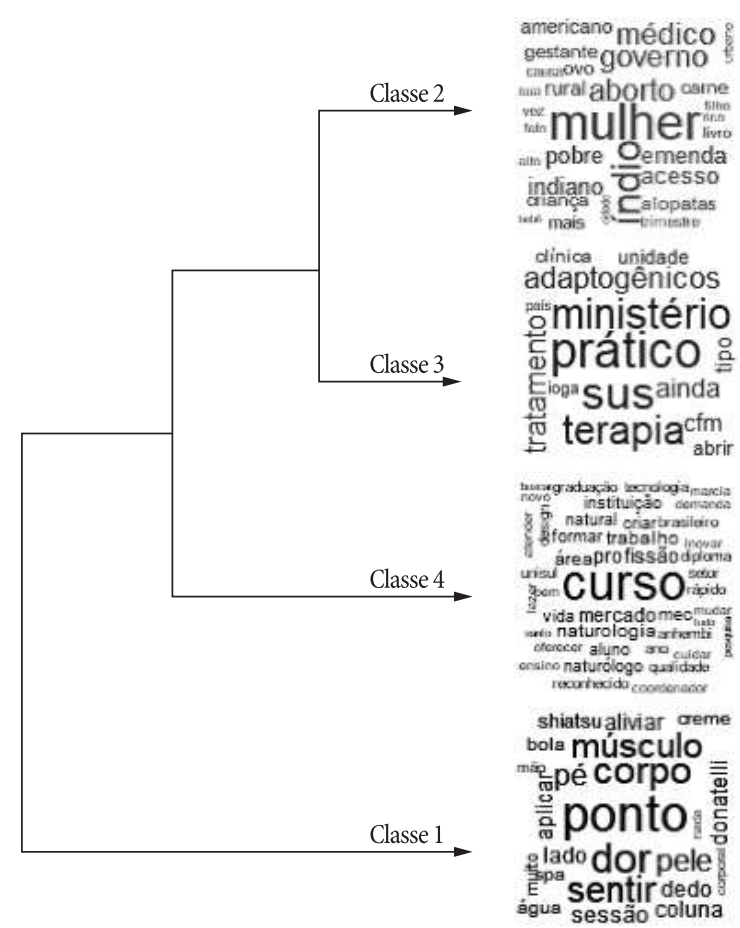

Fonte: Desenvolvido pelos autores. 2018 
Conforme apresentado na figura 01 , a distribuição foi bastante proporcional, destacando-se os corpus de apresentação da naturologia como um curso acadêmico, que exige uma formação profissional. Assim, textos relativos à regularização de curso de naturologia junto ao Ministério da Educação (MEC), tendências do mercado de trabalho para o naturólogo e perfil esperado deste profissional, foram às informações mais apresentadas até o primeiro semestre de 2018.

$\mathrm{O}$ aspecto seguinte, de regulamentação profissional, envolveu a atuação prática do naturólogo, principalmente na participação do profissional junto ao Sistema Único de Saúde (SUS). As normas da política nacional de práticas integrativas e complementares (PNPIC) também abordadas dentro do contexto estudado.

O terceiro ponto mais observado foi a aplicação da naturologia no dia a dia do leitor, desse modo, textos que relatavam e buscavam ensinar pontos de massagem para o alivio da dor, principalmente para o tratamento de dores musculares, foram bastantes divulgados. E, o estímulo em pontos de pressão principalmente no microssistema do pé também foram subtemas bastante abordados.

Por último, e com uma frequência bem menor que os demais pontos apresentados, houve o relato da naturologia direcionada a públicos específicos, sendo a mulher, em estado gravídico, o público mais focado. Além disso, a atenção da naturologia por alguns profissionais, especialmente o médico e o fisioterapeuta, também estiveram presentes nesta subclasse.

Em geral, as palavras mais bem representadas ao longo de todo o corpus, identificam necessidade de trazer mais informação ao leitor acerca de cursos na área, massagens, técnicas e os aspectos científicos da naturologia. Constatou-se que os aspectos científicos, ainda são muito questionados nestas publicações, em especial quando o jornal/revista convida um profissional que não é um naturólogo para falar sobre o assunto, mas que apresenta seu posicionamento sobre os temas e técnicas abordados. A nuvem de palavras apresenta aquelas de maior representação do corpus, conforme exposto na figura 2.

Figura 2 - Nuvem de palavras associadas à naturologia em jornais e revistas não acadêmicas do Brasil. 2018.

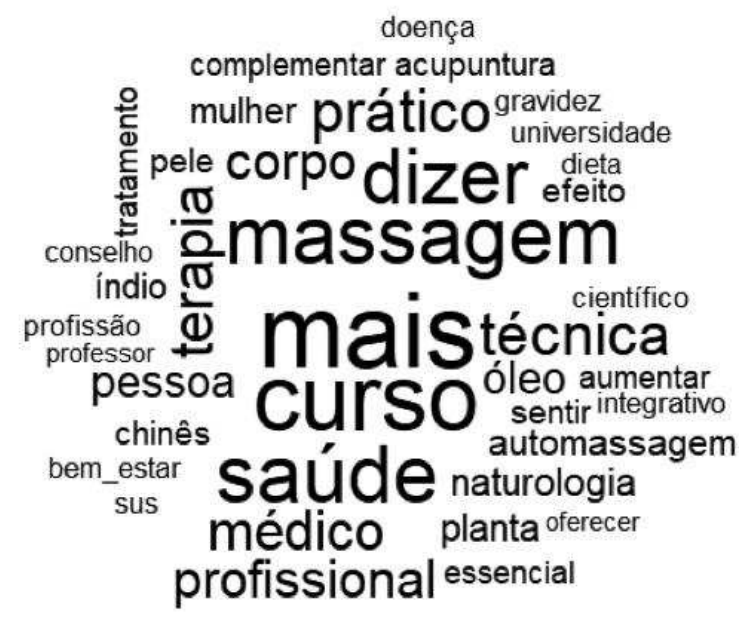

Fonte: Desenvolvido pelos autores. 2018

Mais do que observar o conteúdo do corpus, se fez pertinente observar como as palavras se relacionam, e esta parte da analise nos permitiu identificar que existe claramente um aspecto ainda muito negativo sobre como a naturologia é abordada nas revistas e jornais não acadêmicos no Brasil. Conforme apresentado na figura 3 . 
Figura 3 - Árvore máxima associada à naturologia em jornais e revistas não acadêmicas do Brasil. 2018.

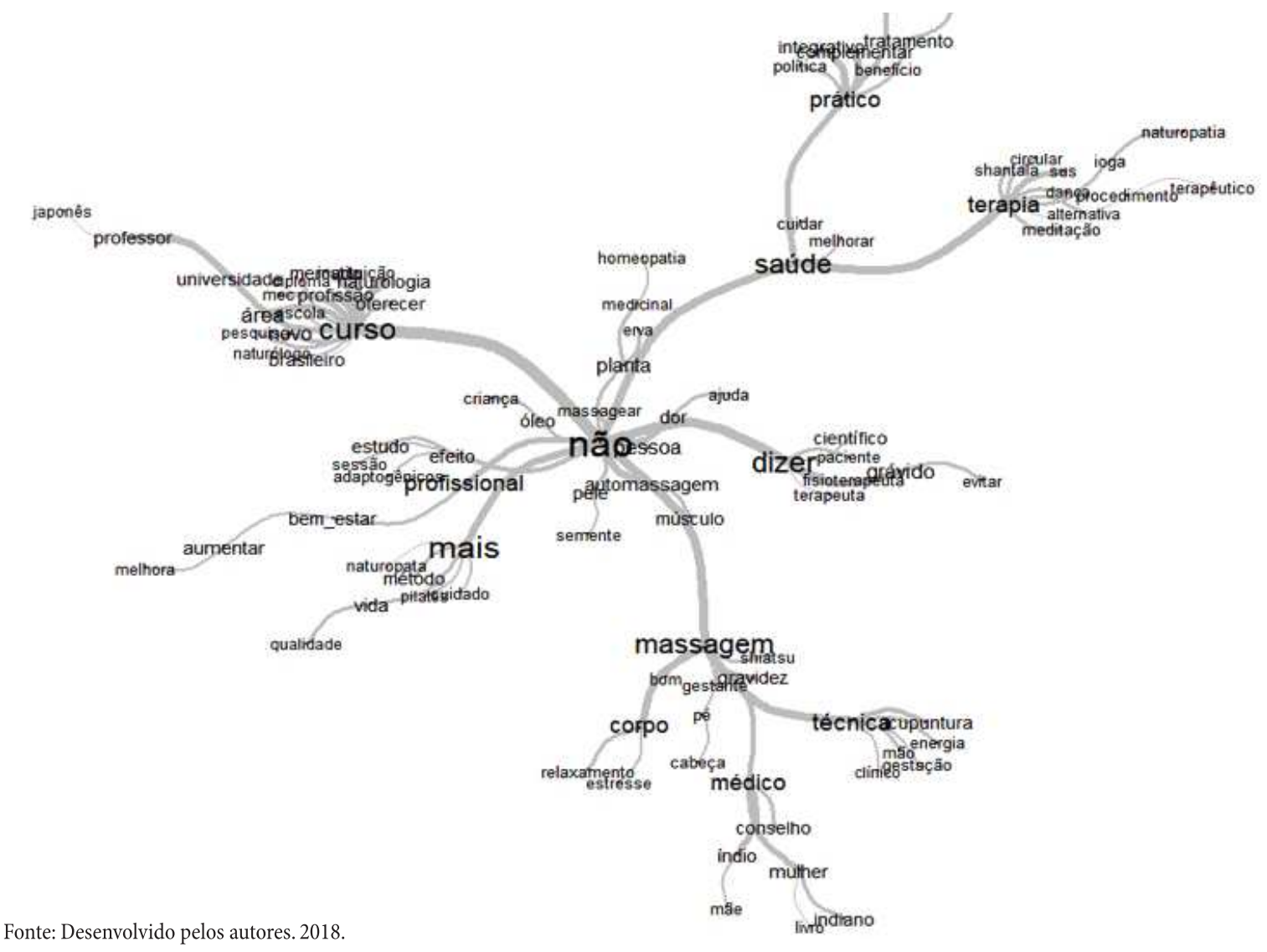

Assim, conforme observado na análise gráfica de especificidade os textos ainda apresentam a naturologia com vários aspectos negativos, o não como palavra central ainda demonstra e denuncia a resistência social, e jornalística, em reconhecer a mesma como uma prática efetiva de cuidado.

Assim, com base em nossos achados podemos inferir que o jornalismo brasileiro e suas produções refletem e moldam a forma de pensar da sociedade. Acredita-se que as produções não acadêmicas no Brasil sejam ainda uma fonte de resistência ao naturologia pelo público em geral, o que pode colaborar para o descrédito da população.

Ainda que haja aspectos positivos e louváveis, oriundos da mídia, como a presença de textos que divulgam a naturologia enquanto ciência com bases acadêmicas e regulamentada pelo governo, há ainda muita divulgação seguida de questionamento das próprias práticas de naturologia. É preciso que jornalistas e editores estejam cientes da sua função social e busquem produzir textos positivos, ou pelos menos neutros das ciências da naturologia. É preciso ainda que, nos textos apresentados ao público, sejam convidados como entrevistados não somente profissionais de outras áreas, mas sim, que os próprios naturólogos sejam convidados a apresentar seus achados.

Espera-se que este trabalho possa contribuir para a sociedade em geral, e que também sirva de ponto de alerta para que naturólogos possam compreendam sua representação junto às mídias não acadêmicas nacionais e que estejam cientes do poder destes meios de comunicação em massa.

\section{DISCUSSÃO}

Ao longo dos últimos anos muito se tem avançado na percepção da naturologia como uma ciência que contém princípios técnicos científicos específicos, com um corpo específico de conhecimentos filosóficos e científicos que moldam suas práticas. E, em nosso país, o avanço das normas e portarias do governo parece ter colaborado para o aumento da percepção científica desta ciência, porém, ainda é preciso avançar, principalmente na divulgação e reconhecimento junto à sociedade não acadêmica. 
No contexto atual, é importante destacar que se tornou muito difícil fazer distinções clássicas entre comunicações interpessoais, organizacionais e de massa, pois a mídia social e os grandes meios de transmissão se conectam de forma cada vez mais complexa e o conteúdo abordado pode fluir de uma rede hiperpessoal para um fórum maior com apenas um clique no mouse. Por outro lado, torna-se cada vez mais claro que todos os eventos também são ocorrências de mídia, na medida em que o que importa tem tanto a ver com a forma como eles são cobertos e a maneira como impactam os fluxos discursivos, quanto com o que realmente acontece no mundo real ${ }^{12}$.

É preciso que a sociedade não acadêmica, e seus jornalistas, compreendam que já existem muitas pesquisas sobre os benefícios da naturologia, não somente como uma prática complementar, mas como uma prática principal de cuidado.

Estudo clínico ${ }^{12}$, mostra que após dois meses de intervenção com práticas de naturologia, clientes diabéticos apresentaram resultados significativos nos níveis de glicose no sangue em jejum e pós-prandial, e, melhora foi observada no Índice de Massa Corporal (IMC). E, outro estudo longitudinal identificou que após o tratamento acompanhando durante 1 ano, com aplicação de massagem, argiloterpaia, águas termais, e práticas regulares de yoga como yogasanas e pranayamas, estas práticas estimularam o sistema imunológico, e promoveram melhorias significativas nos níveis de dor e na diminuição da incapacidade de pacientes com artrite reumatóide ${ }^{14}$.

Entretanto, é preciso que não somente mais pesquisas sejam realizadas, mas que estes estudos sejam conduzidos sob métodos aceitos atualmente, e que as limitações e variáveis confundidoras sejam apresentadas, pois esta clareza de métodos tenderá a colaborar para o aumento da percepção positiva da comunidade geral ${ }^{15}$.

É necessário que profissionais de saúde, mesmo os não naturólogos, sejam capazes de compreender os princípios da naturologia antes de emitir notas sobre as mesmas. Afim de não publicarem opiniões de puro cunho pessoal e sem conhecimento de causa.
Destaca-se que em alguns países, como na Alemanha, desde 2002, estudantes de medicina são requisitados a compreender, e a também explicar os princípios básicos da naturologia e os usos mais comuns das terapias complementares de saúde ${ }^{16}$. Mesmo que não exerçam a naturologia como especialidade médica.

Porém, o que se percebe é que no Brasil, o uso do termo holismo, tão comum nas práticas de naturologia, é ainda visto com receio, devido a tantas banalizações e distorções holísticas. Assim, é preciso a mudança de foco, não mais a doença, mas sim o indivíduo ${ }^{17}$.

Em nossa pesquisa de análise do corpus nacional sobre o tema, além de temas como massagem, foi possível identificar muitos textos que destacavam a ação das plantas, como produtos terapêuticos.

É preciso destacar que a própria história da humanidade entrelaça-se com o consumo de plantas com fins terapêuticos. Ainda, a comunidade acadêmica, defende em sua maioria que as plantas são essenciais para a existência humana. Resultados dos últimos estudos mostram a importância dos antioxidantes para a saúde humana, sendo estes antioxidades amplamente presentes nas plantas recomendadas ao consumo humano ${ }^{18}$.

Outro ponto destacado, e fortemente presente nas publicações com vistas a práticas que os leitores mesmo podiam executar eram a ativação de pontos de pressão. E hoje, já é bem aceito também que a massagem terapêutica oferece muitos benefícios à saúde, com um número crescente de estudos descobrindo que ela tem valor no controle do estresse, na redução da dor e na superação de limitações físicas ${ }^{19}$.

É preciso que a sociedade em geral, bem como os profissionais de jornalismo, mídia e editoração compreendam que existem princípios científicos, bem estabelecidos, que sustentam o processo de pensamento crítico da naturologia, pois não há mais motivos para estas comprovações serem escondidas do público não acadêmico.

É pertinente que a mídia, que também é parte intrínseca da sociedade, compreenda que a carga ideológica inserida no discurso midiático pode se manifestar de diversas e relevantes maneiras, influenciando os padrões de consumo humano. E que compreendam a responsabilidade desta carga divulgacional ${ }^{20}$. 
A invisibilidade midiática de algumas temáticas é um verdadeiro obstáculo ao direito de comunicação. De modo semelhante, a não divulgação de alguns temas de saúde tende a subdimensionar as discussões políticas, econômicas e sociais desses problemas, constituindo-se um entrave à cidadania e à garantia do direito à saúde. Assim, apesar da íntima interface de articulação da comunicação e saúde; a potencialidade dessas relações deve ser melhor explorada. Faz-se necessário uma dinâmica midiática coerente com o destaque das principais necessidades de saúde da população e que potencialize a comunicação como propulsora do direito à saúde dos seus leitores ${ }^{1}$.

\section{CONCLUSÃO}

A mídia jornalística brasileira contribuiu para a divulgação da naturologia como uma ciência acadêmica ao longo dos últimos anos, especialmente entre os anos de 2007 e 2008, porém, as publicações em

\section{CONFLITOS DE INTERESSE}

Declaram não haver.

\section{FONTES DE FINANCIAMENTO}

Declaram não haver.

\section{REFERÊNCIAS}

1. EMERICH, Tatiana Breder et al. Necessidades de saúde e direito à comunicação em tempos de midiatização. FIOCRUZ. RECIIS - Rev Eletron Comun Inf Inov Saúde. 2016 out.-dez.; 10(4) 2016.

2. SABBAG, Silvia Helena Fabbri et al. A naturologia no Brasil avanços e desafios. Cadernos de Naturologia e Terapias Complementares, v. 2, n. 2, p. 11-31,2013

3. DE BARROS, Nelson Filice; LEITE-MOR, Ana Cláudia Moraes Barros. Naturologia e a emergência de novas perspectivas na saúde. Cadernos Acadêmicos, v. 3, n. 2, p. 2-15, 2011.

4. DE OLIVEIRA RODRIGUES, Daniel Maurício; HELLMANN, Fernando; SANCHES, Nathália Martins Pereira. A naturologia e a interface com as racionalidades médicas. Cadernos Acadêmicos, $v$. 3, n. 1, p. 24-36, 2011.

5. RIBEIRO, Thaís Cristina Duarte et al. A contribuição da Naturologia para a implementação da política nacional de práticas integrativas e complementares na concepção dos naturólogos que atuam no Sistema Único de Saúde. Cadernos de Naturologia e Terapias Complementares, v. 6, n. 11, p. 21-32, 2017.

6. CANO-ORÓN, Lorena. Correlación entre las búsquedas sobre terapias complementarias en Google y su uso por parte de la población española. Panace, v. 17, n. 44, 2016. geral ainda focam em aspectos negativos e posicionam esta ciência com muitos pontos questionáveis e por vezes até não os recomenda.

É preciso que a mídia exerça sua função de informar, mas de informar de maneira clara e com menor carga de julgamento possível, deixando então que o leitor/consumidor decida por si quanto ao conteúdo exposto.

Convocamos mais pesquisadores a utilizarem dos achados ora aqui apresentados e propagarem os achados aqui apresentados, acompanhar as representações sociais na mídia e cobrar destes órgãos o direito a informação clara e ampliada.

Ainda, convidamos pesquisadores a ações de intervenção nos pontos aqui identificados, a fim de que seja dada a naturologia e seus profissionais, uma exposição positiva, ou pelo menos neutra, mas que os princípios da mesma sejam divulgados com maior abrangência para o público não acadêmico.
7. COSTA, Midiã Ribeiro. A influência da mídia televisiva nas escolhas alimentares das crianças e na obesidade infantil: uma revisão da literatura. Trabalho de conclusão de curso. Uniceub. 2017.

8. SÁ-SILVA, J.R.; ALMEIDA, C. D.; GUINDANI, J. F. Pesquisa documental: pistas teóricas e metodológicas. Revista Brasileira de História \& Ciências Sociais, v. 1, n. 1, Julho de 2009.

9. SCOTT, John. A matter of record: Documentary sources in social research. John Wiley \& Sons, 2014.

10. CAMARGO, B. V.; JUSTO, A. M. IRAMUTEQ: Um Software Gratuito para Análise de Dados Textuais. Temas em Psicologia, v. 21, n. 2, p. 513-518, 2013.

11. MOSCOVICI, S. Representações sociais: investigações em psicologia social. $9^{\circ}$ ed. Petrópolis: Vozes, 2012.

12. JENKINS, Henry; KALINKE, Priscila; ROCHA, Anderson. Convergência e conexão são o que impulsiona a mídia agora Intercom-Revista Brasileira de Ciências da Comunicação, v. 39, n 1,2016 .

13. NAIR, Rukamani et al. Effect of two months naturopathy treatment in non-insulin dependent diabetes mellitus patients. Int J Sci Res IJSR, v. 5, p. 290-93, 2016. 
14. NAIR, Rukamani et al. Improvement in Physical Parameters of Rheumatoid Arthritis Patients by Naturopathy and Yoga. International Journal of Multidisciplinary Approach \& Studies, v. 1, n. 4, p. 132-146, 2014

15. ARENTZ, Susan. Developing intellectual capacity in Naturopathy and Herbal Medicine practice. Australian Journal of Herbal and Naturopathic Medicine, v. 30, n. 1, 2018.

16. VALENTINI, J. et al. “Critical discussion should be encouraged!"-a qualitative analysis of medical students' evaluation of a complementary medicine course. Deutsche medizinische Wochenschrift (1946), v. 143, n. 14, p. el25-e130, 2018.
17. DE OLIVEIRA RODRIGUES, Daniel Maurício et al. Afinal, Naturologia e Naturologia são coisas distintas ou similares? Cadernos de Naturologia e Terapias Complementares, v. 6, n. 10, p. 9-12, 2017.

18. SELAMOGLU, Z. Traditional Medicine \& Clinical Naturopathy Pharm Res, v. 16, p. 92-98, 2018.

19. COTTINGHAM, Phillip et al. The Characteristics, Experiences and Perceptions of Registered Massage Therapists in New Zealand: Results from a National Survey of Practitioners. International journal of therapeutic massage \& bodywork, v. 11, n. 2, p. 11, 2018.

20. LOPEZ, Debora Cristina; DITTRICH, Ivo José. A palavra como signo ideológico no discurso jornalístico. Bocc, v. 26, 2017. 